\title{
Heterogenous Effect of Risk Factors on Breast Cancer across the Breast Density Categories in a Korean Screening Population
}

\author{
Boyoung Park ${ }^{1, * \mathbb{D}}$, Se-Eun Lim ${ }^{1}$, HyoJin Ahn ${ }^{1}$, Junghyun Yoon ${ }^{2}$ and Yun Su Choi ${ }^{3}$ \\ 1 Department of Medicine, Hanyang University College of Medicine, Seoul 04763, Korea; \\ seeunlim1022@gmail.com (S.-E.L.); deoldsong@gmail.com (H.A.) \\ 2 Graduate School of Public Health, Hanyang University, Seoul 04763, Korea; cumyluceat@hanyang.ac.kr \\ 3 Department of Preventive Medicine, Hanyang University College of Medicine, Seoul 04763, Korea; \\ 90dbstn@naver.com \\ * Correspondence: hayejine@hanyang.ac.kr; Tel.: +82-2-2220-0682
}

Received: 23 April 2020; Accepted: 26 May 2020; Published: 28 May 2020

\begin{abstract}
We evaluated the heterogeneity of the effect of known risk factors on breast cancer development based on breast density by using the Breast Imaging-Reporting and Data System (BI-RADS). In total, 4,898,880 women, aged 40-74 years, who participated in the national breast cancer screening program in 2009-2010 were followed up to December 2018. Increased age showed a heterogeneous association with breast cancer (1-year hazard ratio $(\mathrm{HR})=0.92,1.00$ (reference), 1.03, and 1.03 in women with BI-RADS density category 1, 2,3, and 4, respectively; P-heterogeneity $<0.001$ ). More advanced age at menopause increased breast cancer risk in all BI-RADS categories. This was more prominent in women with BI-RADS density category 1 but less prominent in women in other BI-RADS categories (P-heterogeneity $=0.009$ ). In postmenopausal women, a family history of breast cancer, body mass index $\geq 25 \mathrm{~kg} / \mathrm{m}^{2}$, and smoking showed a heterogeneous association with breast cancer across all BI-RADS categories. Other risk factors including age at menarche, menopause, hormone replacement therapy after menopause, oral contraceptive use, and alcohol consumption did not show a heterogeneous association with breast cancer across the BI-RADS categories. Several known risk factors of breast cancer had a heterogeneous effect on breast cancer development across breast density categories, especially in postmenopausal women.
\end{abstract}

Keywords: breast cancer; Breast Imaging-Reporting and Data System; breast density; risk factors; heterogeneous effect

\section{Introduction}

Breast cancer is the most common cancer in women worldwide, accounting for $24.4 \%$ of all types of cancer; moreover, breast cancer is the leading cause of cancer death in women [1]. In Asian countries, breast cancer incidence is lower; however, it has rapidly increased over the past decades. Changes in demographic factors associated with social and economic development, including lifestyle and reproductive risk factors, increase in breast cancer screening, and awareness in the region, have been attributed to this trend [1,2].

Breast density is a non-modifiable risk factor for breast cancer and can be identified through breast cancer screening using a mammogram. High mammographic density increases the risk of breast cancer; women with a breast tissue density $\geq 75 \%$ have a $4-5$ times higher risk compared with those with a density $<5 \%[3,4]$. The effect of mammographic density on breast cancer risk has been investigated previously. Studies have suggested that breast density is associated with risk factors of breast cancer such as age, reproductive factors, and body mass index (BMI) [5-7]. Other studies 
investigated whether there is an interaction effect between breast density and the known risk factors on breast cancer. It has been shown that the effect of breast density can be increased or decreased by family history, reproductive factors, behavioral factors, and body mass index (BMI) [8-13].

Most previous studies that investigated the interaction effect between breast density and other risk factors on breast cancer development considered other risk factors as effect modifiers and breast density as an independent variable of interest [8-13]. Considering a preventive approach in treatment, whether the effects of other breast cancer risk factors differ by breast density [14], needs to be assessed. In addition, the subjects of these studies included only women in the US, Japan, or France. Considering the differences in breast cancer epidemiology between countries, the interaction effect between breast density and other risk factors needs to be assessed in other populations.

In Asian countries, known breast cancer risk factors are less prevalent; however, the prevalence of dense breasts is high [6] and screening mammography programs have only started in some countries [15]. Furthermore, the evaluation of the relationship of mammographic density and breast cancer risk, considering its interaction with other risk factors, has been limited. Therefore, in this study, we comprehensively examined the interaction effect between breast density, which is measured during mammographic screening, and various risk factors on breast cancer development by assessing the heterogeneity of the effect of these risk factors based on breast density in a large East Asian population for individualized risk assessment within the screening setting.

\section{Results}

Table 1 presents the distribution of breast cancer risk factors in participants with or without incident breast cancer according to the Breast Imaging-Reporting and Data System (BI-RADS) density category. Among 5,038,851 subjects, after excluding 141,345 women with missing BI-RADS density or with breast implants, $14.8 \%, 23.5 \%, 37.8 \%$, and $23.9 \%$ of breast cancer incident cases were classified as BI-RADS density category $1,2,3$, and 4 , respectively. The corresponding values in controls were $27.3 \%$, $27.4 \%, 30.1 \%$ and $15.2 \%$.

The associations between risk factors and the development of breast cancer stratified by breast density, and heterogeneity of the association between BI-RADS categories are presented in Table 2 . For participants with BI-RADS density category 1 , increased age was associated with a decreased risk of breast cancer $(\mathrm{HR}=0.92 ; 95 \% \mathrm{CI}=0.90-0.93)$; no association was observed in participants with BI-RADS category 2 and an increased association was observed in participants with BI-RADS category 3 and $4(\mathrm{HR}=1.03 ; 95 \% \mathrm{CI}=1.01-1.04, \mathrm{HR}=1.03 ; 95 \% \mathrm{CI}=1.01-1.06$, respectively; P-heterogeneity between the four groups $<0.001$ ). Advanced age at menopause increased breast cancer risk in all BI-RADS categories; this was significantly more prominent in participants with BI-RADS density category 1 (age $>51$ years at menopause, $\mathrm{HR}=1.46 ; 95 \% \mathrm{CI}=1.37-1.56$ ) and less prominent in women with other BI-RAD density categories $(\mathrm{HR}=1.28,95 \% \mathrm{CI}=1.21-1.35 ; \mathrm{HR}=1.33,95 \% \mathrm{CI}=1.25-1.41$; and $\mathrm{HR}=1.24,95 \% \mathrm{CI}=1.12-1.38$ for BI-RADS 2, 3, and 4, respectively; P-heterogeneity $=0.009$ ). Other risk factors did not show a heterogeneous association with breast cancer across BI-RADS categories. Advanced age at menarche, post-menopausal status, and no use of hormone replacement therapy after menopause decreased breast cancer risk; a family history of breast cancer increased the risk irrespective of density category. Menopause at the age of 50-51 years compared with that at $\leq 49$ years, use of hormone replacement therapy after menopause for $\geq 1$ year compared with that for $<1$ year, and higher BMI increased the risk of breast cancer in all density categories.

When stratified by menopausal status, a heterogeneous effect of each risk factor on breast cancer based on the four density categories was not observed in premenopausal participants (Table 3). Despite the lack of heterogeneity, there was no association of BMI with breast cancer in participants with BI-RADS category 1 or 2 . However, as BMI increased, the risk of breast cancer was also found to increase; this was more prominent in women with BI-RADS density category 4. 
Table 1. Distribution of risk factors of breast cancer in subjects with or without incident breast cancer by mammographic density.

\begin{tabular}{|c|c|c|c|c|c|c|c|c|c|c|c|c|c|c|c|c|c|c|c|c|}
\hline \multirow{2}{*}{ Risk Factor } & \multicolumn{4}{|c|}{ Total * } & \multicolumn{4}{|c|}{ BI-RADS 1} & \multicolumn{4}{|c|}{ BI-RADS 2} & \multicolumn{4}{|c|}{ BI-RADS 3} & \multicolumn{4}{|c|}{ BI-RADS 4} \\
\hline & \multicolumn{2}{|c|}{$\begin{array}{c}\text { Case } \\
(n=55,538)\end{array}$} & \multicolumn{2}{|c|}{$\begin{array}{c}\text { Control } \\
(n=4,983,313) \\
\end{array}$} & \multicolumn{2}{|c|}{$\begin{array}{c}\text { Case } \\
(n=8002)\end{array}$} & \multicolumn{2}{|c|}{$\begin{array}{c}\text { Control } \\
(n=1,321,826) \\
\end{array}$} & \multicolumn{2}{|c|}{$\begin{array}{c}\text { Case } \\
(n=12,734)\end{array}$} & \multicolumn{2}{|c|}{$\begin{array}{c}\text { Control } \\
(n=1,328,735) \\
\end{array}$} & \multicolumn{2}{|c|}{$\begin{array}{c}\text { Case } \\
(n=20,478)\end{array}$} & \multicolumn{2}{|c|}{$\begin{array}{c}\text { Control } \\
(n=1, \mathbf{4 5 6 , 4 1 9 )} \\
\end{array}$} & \multicolumn{2}{|c|}{$\begin{array}{c}\text { Case } \\
(n=12,950)\end{array}$} & \multicolumn{2}{|c|}{$\begin{array}{c}\text { Control } \\
(n=736,362)\end{array}$} \\
\hline Age, Mean (SD) & \multicolumn{2}{|c|}{$51.42(8.61)$} & \multicolumn{2}{|c|}{$53.99(9.55)$} & \multicolumn{2}{|c|}{$58.18(9.02)$} & \multicolumn{2}{|c|}{$59.16(8.79)$} & \multicolumn{2}{|c|}{$54.69(8.53)$} & $55.37(8$ & .82) & 49.73( & $(49.68)$ & 50.05( & & 46.68 & (5.96) & 46.93( & 27) \\
\hline $40-44$ & 14,812 & $26.7 \%$ & $1,021,194$ & $20.5 \%$ & 799 & $10.0 \%$ & 80,134 & $6.1 \%$ & 1912 & $15.0 \%$ & 181,841 & $13.7 \%$ & 6061 & $29.6 \%$ & 422,209 & $29.0 \%$ & 5764 & $44.5 \%$ & 320,782 & $43.6 \%$ \\
\hline $45-49$ & 10,816 & $19.5 \%$ & 767,644 & $15.4 \%$ & 671 & $8.4 \%$ & 78,169 & $5.9 \%$ & 1655 & $13.0 \%$ & 163,811 & $12.3 \%$ & 4792 & $23.4 \%$ & 316,858 & $21.8 \%$ & 3516 & $27.2 \%$ & 192,962 & $26.2 \%$ \\
\hline $50-54$ & 12,057 & $21.7 \%$ & $1,048,644$ & $21.0 \%$ & 1322 & $16.5 \%$ & 195,289 & $14.8 \%$ & 2979 & $23.4 \%$ & 312,757 & $23.5 \%$ & 4956 & $24.2 \%$ & 368,041 & $25.3 \%$ & 2481 & $19.2 \%$ & 146,180 & $19.9 \%$ \\
\hline $55-59$ & 6570 & $11.8 \%$ & 629,573 & $12.6 \%$ & 1307 & $16.3 \%$ & 193,944 & $14.7 \%$ & 2226 & $17.5 \%$ & 220,726 & $16.6 \%$ & 2212 & $10.8 \%$ & 156,762 & $10.8 \%$ & 639 & $4.9 \%$ & 39,648 & $5.4 \%$ \\
\hline $60-64$ & 6051 & $10.9 \%$ & 661,677 & $13.3 \%$ & 1741 & $21.8 \%$ & 278,030 & $21.0 \%$ & 2218 & $17.4 \%$ & 221,162 & $16.6 \%$ & 1515 & $7.4 \%$ & 114,718 & $7.9 \%$ & 366 & $2.8 \%$ & 23,110 & $3.1 \%$ \\
\hline $65-69$ & 3252 & $5.9 \%$ & 460,749 & $9.2 \%$ & 1204 & $15.0 \%$ & 245,385 & $18.6 \%$ & 1124 & $8.8 \%$ & 135,147 & $10.2 \%$ & 676 & $3.3 \%$ & 51,565 & $3.5 \%$ & 137 & $1.1 \%$ & 9202 & $1.2 \%$ \\
\hline $70-74$ & 1980 & $3.6 \%$ & 393,832 & $7.9 \%$ & 958 & $12.0 \%$ & 250,875 & $19.0 \%$ & 620 & $4.9 \%$ & 93,291 & $7.0 \%$ & 266 & $1.3 \%$ & 26,266 & $1.8 \%$ & 47 & $0.4 \%$ & 4478 & $8.6 \%$ \\
\hline Age at Menarche & & & & & & & & & & & & & & & & & & & & \\
\hline$\leq 15$ & 29,644 & $53.4 \%$ & $2,225,369$ & $44.7 \%$ & 3070 & $38.4 \%$ & 405,086 & $30.6 \%$ & 5907 & $46.4 \%$ & 550,020 & $41.4 \%$ & 11,810 & $57.7 \%$ & 776,838 & $53.3 \%$ & 8256 & $63.8 \%$ & 447,264 & $60.7 \%$ \\
\hline $16-17$ & 16,767 & $30.2 \%$ & $1,674,284$ & $33.6 \%$ & 2968 & $37.1 \%$ & 512,261 & $38.8 \%$ & 4279 & $33.6 \%$ & 468,669 & & 5855 & $28.6 \%$ & 443,922 & $30.5 \%$ & 3253 & $25.1 \%$ & 200,494 & $27.2 \%$ \\
\hline$>17$ & 7178 & $12.9 \%$ & 909,998 & $18.3 \%$ & 1679 & $21.0 \%$ & 358,948 & $27.2 \%$ & 2080 & $16.3 \%$ & 263,207 & $19.8 \%$ & 2171 & $10.6 \%$ & 187,849 & $12.9 \%$ & 1019 & $7.9 \%$ & 68,442 & $9.3 \%$ \\
\hline Missing & 1949 & $3.5 \%$ & 173,662 & $3.5 \%$ & 285 & $3.6 \%$ & 45,531 & $3.4 \%$ & 468 & $3.7 \%$ & 46,839 & $3.5 \%$ & 642 & $3.1 \%$ & 47,810 & $3.3 \%$ & 422 & $3.3 \%$ & 20,162 & $2.7 \%$ \\
\hline Menopausal Statu & & & & & & & & & & & & & & & & & & & & \\
\hline Premenopause & 31,145 & $56.1 \%$ & $2,211,172$ & $44.4 \%$ & 2214 & $27.7 \%$ & 264,153 & $20.0 \%$ & 4951 & $38.9 \%$ & 467,950 & $35.2 \%$ & 13,005 & $63.5 \%$ & 871,198 & $59.8 \%$ & 10,389 & $80.2 \%$ & 565,379 & $76.8 \%$ \\
\hline Postmenopause & 23,406 & $42.1 \%$ & $2,687,044$ & $53.9 \%$ & 5652 & $70.6 \%$ & $1,037,607$ & $78.5 \%$ & 7531 & $59.1 \%$ & 835,310 & $62.9 \%$ & 7121 & $34.8 \%$ & 561,211 & $38.5 \%$ & 2358 & $18.2 \%$ & 160,705 & $21.8 \%$ \\
\hline Unknown & 987 & $1.8 \%$ & 85,097 & $1.7 \%$ & 136 & $1.7 \%$ & 20,066 & $1.5 \%$ & 252 & $2.0 \%$ & 25,475 & $1.9 \%$ & 352 & $1.7 \%$ & 24,010 & $1.6 \%$ & 203 & $1.6 \%$ & 10,278 & $1.4 \%$ \\
\hline Age at Menopaus & & & & & & & & & & & & & & & & & & & & \\
\hline Premenopause & 31,145 & $56.1 \%$ & $2,211,172$ & $44.4 \%$ & 2214 & $27.7 \%$ & 264,153 & $20.0 \%$ & 4951 & $38.9 \%$ & 467,950 & $35.2 \%$ & 13,005 & $63.5 \%$ & 871,198 & $59.8 \%$ & 10,389 & $80.2 \%$ & 565,379 & $76.8 \%$ \\
\hline$\leq 49$ & 6240 & $11.2 \%$ & 849,192 & $17.0 \%$ & 1608 & $20.1 \%$ & 359,477 & $27.2 \%$ & 2038 & $16.0 \%$ & 252,046 & $19.0 \%$ & 1765 & $8.6 \%$ & 161,408 & $11.1 \%$ & 654 & $5.1 \%$ & 48,709 & $6.6 \%$ \\
\hline$\leq 51$ & 6219 & $11.2 \%$ & 739,689 & $14.8 \%$ & 1504 & $18.8 \%$ & 289,118 & $21.9 \%$ & 1987 & $15.6 \%$ & 229,085 & $17.2 \%$ & 1906 & $9.3 \%$ & 151,566 & $10.4 \%$ & 623 & $4.8 \%$ & 43,333 & $5.9 \%$ \\
\hline$>51$ & 9157 & $16.5 \%$ & 919,834 & $18.5 \%$ & 2256 & $28.2 \%$ & 342,243 & $25.9 \%$ & 3076 & $24.2 \%$ & 303,884 & $22.9 \%$ & 2783 & $13.6 \%$ & 197,492 & $13.6 \%$ & 797 & $6.2 \%$ & 49,071 & $6.7 \%$ \\
\hline Unknown & 2777 & $5.0 \%$ & 263,426 & $5.3 \%$ & 420 & $5.2 \%$ & 66,835 & $5.1 \%$ & 682 & $5.4 \%$ & 75,770 & $5.7 \%$ & 1019 & $5.0 \%$ & 74,755 & $5.1 \%$ & 487 & $3.8 \%$ & 29,870 & $4.1 \%$ \\
\hline Hormone Replace & nt Thera & & & & & & & & & & & & & & & & & & & \\
\hline No Usage & 17,409 & $31.3 \%$ & $2,142,206$ & $43.0 \%$ & 4518 & $56.5 \%$ & 872,119 & $66.0 \%$ & 5677 & $44.6 \%$ & 653,482 & $49.2 \%$ & 5014 & $24.5 \%$ & 421,259 & $28.9 \%$ & 1649 & $12.7 \%$ & 119,807 & $16.3 \%$ \\
\hline$<1$ Year & 3764 & $6.8 \%$ & 354,520 & $7.1 \%$ & 709 & $8.9 \%$ & 103,980 & $7.9 \%$ & 1169 & & 119,573 & $9.0 \%$ & 1326 & $6.5 \%$ & 93,767 & $6.4 \%$ & 436 & $3.4 \%$ & 27,864 & $3.8 \%$ \\
\hline$\geq 1$ Year & 2089 & $3.8 \%$ & 175,189 & $3.5 \%$ & 394 & $4.9 \%$ & 56,850 & $4.3 \%$ & 640 & $5.0 \%$ & 57,935 & $4.4 \%$ & 744 & $3.6 \%$ & 43,006 & $3.0 \%$ & 257 & $2.0 \%$ & 12,118 & $1.6 \%$ \\
\hline Premenopause & 31,145 & $56.1 \%$ & $2,211,172$ & $44.4 \%$ & 2214 & $27.7 \%$ & 264,153 & $20.0 \%$ & 4951 & $38.9 \%$ & 467,950 & $35.2 \%$ & 13,005 & $63.5 \%$ & 871,198 & $59.8 \%$ & 10,389 & $80.2 \%$ & 565,379 & $76.8 \%$ \\
\hline Unknown & 1131 & $2.0 \%$ & 100,226 & $2.0 \%$ & 167 & $2.1 \%$ & 24,724 & $1.9 \%$ & 297 & $2.3 \%$ & 29,795 & $2.2 \%$ & 389 & $1.9 \%$ & 27,189 & $1.9 \%$ & 219 & $1.7 \%$ & 11,194 & $1.5 \%$ \\
\hline Oral Contraceptiv & & & & & & & & & & & & & & & & & & & & \\
\hline No Usage & 44,041 & $79.3 \%$ & $3,950,946$ & $79.3 \%$ & 6170 & $77.1 \%$ & $1,035,264$ & $78.3 \%$ & 9860 & $77.4 \%$ & $1,034,792$ & $77.9 \%$ & 16,355 & $79.9 \%$ & $1,167,219$ & $80.1 \%$ & 10,582 & $81.7 \%$ & 604,281 & $82.1 \%$ \\
\hline$<6$ Months & 5231 & $9.4 \%$ & 458,377 & $9.2 \%$ & 766 & $9.6 \%$ & 119,482 & $9.0 \%$ & 1246 & $9.8 \%$ & 127,147 & $9.6 \%$ & 1918 & $9.4 \%$ & 135,007 & $9.3 \%$ & 1176 & $9.1 \%$ & 65,825 & $8.9 \%$ \\
\hline$\geq 6$ Months & 5200 & $9.4 \%$ & 481,640 & $9.7 \%$ & 910 & $11.4 \%$ & 145,296 & $11.0 \%$ & 1370 & $10.8 \%$ & 140,045 & $10.5 \%$ & 1833 & $9.0 \%$ & 128,993 & $8.9 \%$ & 972 & $7.5 \%$ & 54,928 & $7.5 \%$ \\
\hline Unknown & 1066 & $1.9 \%$ & 92,350 & $1.9 \%$ & 156 & $1.9 \%$ & 21,784 & $1.6 \%$ & 258 & $2.0 \%$ & 26,751 & $2.0 \%$ & 372 & $1.8 \%$ & 25,200 & $1.7 \%$ & 220 & $1.7 \%$ & 11,328 & $1.5 \%$ \\
\hline
\end{tabular}


Table 1. Cont.

\begin{tabular}{|c|c|c|c|c|c|c|c|c|c|c|c|c|c|c|c|c|c|c|c|c|}
\hline \multirow{2}{*}{ Risk Factor } & \multicolumn{4}{|c|}{ Total * } & \multicolumn{4}{|c|}{ BI-RADS 1} & \multicolumn{4}{|c|}{ BI-RADS 2} & \multicolumn{4}{|c|}{ BI-RADS 3} & \multicolumn{4}{|c|}{ BI-RADS 4} \\
\hline & \multicolumn{2}{|c|}{$\begin{array}{c}\text { Case } \\
(n=55,538)\end{array}$} & \multicolumn{2}{|c|}{$\begin{array}{c}\text { Control } \\
(n=4,983,313) \\
\end{array}$} & \multicolumn{2}{|c|}{$\begin{array}{c}\text { Case } \\
(n=8002)\end{array}$} & \multicolumn{2}{|c|}{$\begin{array}{c}\text { Control } \\
(n=1,321,826) \\
\end{array}$} & \multicolumn{2}{|c|}{$\begin{array}{c}\text { Case } \\
(n=12,734)\end{array}$} & \multicolumn{2}{|c|}{$\begin{array}{c}\text { Control } \\
(n=1,328,735) \\
\end{array}$} & \multicolumn{2}{|c|}{$\begin{array}{c}\text { Case } \\
(n=20,478)\end{array}$} & \multicolumn{2}{|c|}{$\begin{array}{c}\text { Control } \\
(n=1, \mathbf{4 5 6 , 4 1 9 )} \\
\end{array}$} & \multicolumn{2}{|c|}{$\begin{array}{c}\text { Case } \\
(n=12,950)\end{array}$} & \multicolumn{2}{|c|}{$\begin{array}{c}\text { Control } \\
(n=736,362)\end{array}$} \\
\hline \multicolumn{21}{|c|}{ Family History (in First Degree Relative) } \\
\hline No & 53,744 & $96.8 \%$ & $4,906,815$ & $98.5 \%$ & 7803 & $97.5 \%$ & $1,307,783$ & $98.9 \%$ & 12383 & $97.2 \%$ & $1,308,937$ & $98.5 \%$ & 19,762 & $96.5 \%$ & $1,429,547$ & $98.2 \%$ & 12,450 & $96.1 \%$ & 721,919 & $98.0 \%$ \\
\hline Yes & 1794 & $3.2 \%$ & 76,498 & $1.5 \%$ & 199 & $2.5 \%$ & 14,043 & $1.1 \%$ & 351 & $2.8 \%$ & 19,798 & $1.5 \%$ & 716 & $3.5 \%$ & 26,872 & $1.8 \%$ & 500 & $3.9 \%$ & 14,443 & $2.0 \%$ \\
\hline \multicolumn{21}{|l|}{ BMI $\left(\mathrm{Kg} / \mathrm{m}^{2}\right)$} \\
\hline$<18.5$ & 1170 & $2.1 \%$ & 108,320 & $2.2 \%$ & 71 & $0.9 \%$ & 17,390 & $1.3 \%$ & 130 & $1.0 \%$ & 19,134 & $1.4 \%$ & 396 & $1.9 \%$ & 32,893 & $2.3 \%$ & 556 & $4.3 \%$ & 36,409 & $4.9 \%$ \\
\hline$<23$ & 23,032 & $41.5 \%$ & $1,978,032$ & $39.7 \%$ & 2008 & $25.1 \%$ & 372,024 & $28.1 \%$ & 4107 & $32.3 \%$ & 465,450 & $35.0 \%$ & 9116 & $44.5 \%$ & 673,523 & $46.2 \%$ & 7302 & $56.4 \%$ & 417,550 & $56.7 \%$ \\
\hline$<25$ & 13,483 & $24.3 \%$ & $1,256,999$ & $25.2 \%$ & 1913 & $23.9 \%$ & 338,400 & $25.6 \%$ & 3305 & $26.0 \%$ & 357,089 & $26.9 \%$ & 5225 & $25.5 \%$ & 369,801 & $25.4 \%$ & 2713 & $20.9 \%$ & 155,051 & $21.1 \%$ \\
\hline$>25$ & 17,836 & $32.1 \%$ & $1,638,953$ & $32.9 \%$ & 4008 & $50.1 \%$ & 593,663 & $44.9 \%$ & 5188 & $40.7 \%$ & 486,839 & $36.6 \%$ & 5738 & $28.0 \%$ & 379,964 & $26.1 \%$ & 2372 & $18.3 \%$ & 127,198 & $17.3 \%$ \\
\hline Unknown & 17 & $0.0 \%$ & 1009 & $0.0 \%$ & 2 & $0.0 \%$ & 349 & $0.0 \%$ & 4 & $0.0 \%$ & 223 & $0.0 \%$ & 3 & $0.0 \%$ & 238 & $0.0 \%$ & 7 & $0.1 \%$ & 154 & $0.0 \%$ \\
\hline \multicolumn{21}{|c|}{ Smoking in Lifetime } \\
\hline Never Smoked & 52,452 & $94.4 \%$ & $4,736,003$ & $95.0 \%$ & 7622 & $95.3 \%$ & $1,268,071$ & $95.9 \%$ & 12,091 & $95.0 \%$ & $1,263,989$ & $95.1 \%$ & 19,253 & $94.0 \%$ & $1,377,655$ & $94.6 \%$ & 12,202 & $94.2 \%$ & 695,249 & $94.4 \%$ \\
\hline Ever Smoked & 2795 & $5.0 \%$ & 221,184 & $4.4 \%$ & 329 & $4.1 \%$ & 47,225 & $3.6 \%$ & 580 & $4.6 \%$ & 58,230 & $4.4 \%$ & 1127 & $5.5 \%$ & 71,451 & $4.9 \%$ & 701 & $5.4 \%$ & 38,524 & $5.2 \%$ \\
\hline Missing & 291 & $0.5 \%$ & 26,126 & $0.5 \%$ & 51 & $0.6 \%$ & 6530 & $0.5 \%$ & 63 & $0.5 \%$ & 6516 & $0.5 \%$ & 98 & $0.5 \%$ & 7313 & $0.5 \%$ & 47 & $0.4 \%$ & 2589 & $0.4 \%$ \\
\hline \multicolumn{21}{|c|}{ MeanDays of Drinking per Week during The Last 1 Year } \\
\hline No Drinking & 42,705 & $76.9 \%$ & $3,954,159$ & $79.3 \%$ & 6738 & $84.2 \%$ & $1,135,424$ & $85.9 \%$ & 10,213 & $80.2 \%$ & $1,073,640$ & $80.8 \%$ & 15,355 & $75.0 \%$ & $1,098,610$ & $75.4 \%$ & 9373 & $72.4 \%$ & 536,517 & $72.9 \%$ \\
\hline$\geq 1$ Day & 12,328 & $22.2 \%$ & 984,611 & $19.8 \%$ & 1194 & $14.9 \%$ & 174,844 & $13.2 \%$ & 2387 & $18.7 \%$ & 243,220 & $18.3 \%$ & 4981 & $24.3 \%$ & 347,920 & $23.9 \%$ & 3517 & $27.2 \%$ & 196,675 & $26.7 \%$ \\
\hline Missing & 505 & $0.9 \%$ & 44,543 & $0.9 \%$ & 70 & $0.9 \%$ & 11,558 & $0.9 \%$ & 134 & $1.1 \%$ & 11,875 & $0.9 \%$ & 142 & $0.7 \%$ & 9889 & $0.7 \%$ & 60 & $0.4 \%$ & 3170 & $0.4 \%$ \\
\hline \multicolumn{21}{|c|}{ MeanDays of Physical Activity per Week } \\
\hline No Activity & 29,643 & $53.4 \%$ & $2,782,364$ & $55.8 \%$ & 4703 & $58.8 \%$ & 813,001 & $61.5 \%$ & 7000 & $55.0 \%$ & 743,828 & $56.0 \%$ & 10,564 & $51.6 \%$ & 765,617 & $52.6 \%$ & 6678 & $51.6 \%$ & 383,117 & $52.0 \%$ \\
\hline$\geq 1$ Day & 25,607 & $46.1 \%$ & $2,175,000$ & $43.6 \%$ & 3256 & $40.7 \%$ & 501,918 & $38.0 \%$ & 5665 & $44.5 \%$ & 578,492 & $43.5 \%$ & 9825 & $48.0 \%$ & 684,647 & $47.0 \%$ & 6230 & $48.0 \%$ & 351,149 & $47.7 \%$ \\
\hline Unknown & 288 & $0.5 \%$ & 25,949 & $0.5 \%$ & 43 & $0.5 \%$ & 6907 & $0.5 \%$ & 69 & $0.5 \%$ & 6415 & $0.5 \%$ & 89 & $0.4 \%$ & 6155 & $0.4 \%$ & 42 & $0.3 \%$ & 2096 & $0.3 \%$ \\
\hline
\end{tabular}

Table 2. Association between known risk factors of breast cancer and breast cancer development by breast density, in consideration of their interaction on breast cancer risk.

\begin{tabular}{|c|c|c|c|c|c|c|c|c|c|c|c|c|c|c|c|c|}
\hline \multirow{2}{*}{ Risk Factor } & \multicolumn{3}{|c|}{ Total } & \multicolumn{3}{|c|}{ BI-RADS 1} & \multicolumn{3}{|c|}{ BI-RADS 2} & \multicolumn{3}{|c|}{ BI-RADS 3} & \multicolumn{3}{|c|}{ BI-RADS 4} & \multirow{2}{*}{ P-heterogeneity } \\
\hline & HR * & $95 \%$ CI & $\mathbf{P}$ & HR * & $95 \%$ CI & $p$-value & $\mathrm{HR}^{*}$ & $95 \%$ CI & $\mathbf{P}$ & HR * & $95 \% \mathrm{CI}$ & $\mathbf{P}$ & HR * & $95 \%$ CI & $\mathbf{P}$ & \\
\hline \multicolumn{17}{|l|}{ Age } \\
\hline $\begin{array}{l}1 \text { Year Increment } \\
\text { Age at Menarche }\end{array}$ & 0.93 & $(0.92-0.93)$ & $<0.001$ & 0.92 & $(0.90-0.93)$ & $<0.001$ & 1.00 & $(0.98-1.01)$ & 0.512 & 1.03 & $(1.01-1.04)$ & $<0.001$ & 1.03 & $(1.01-1.06)$ & 0.001 & $<0.001$ \\
\hline$\leq 15$ & 1 & & & 1 & & & 1 & & & 1 & & & 1 & & & \\
\hline $16-17$ & 0.88 & $(0.87-0.9)$ & $<0.001$ & 0.88 & $(0.83-0.92)$ & $<0.001$ & 0.89 & $(0.85-0.92)$ & $<0.001$ & 0.89 & $(0.86-0.92)$ & $<0.001$ & 0.90 & $(0.86-0.94)$ & $<0.001$ & 0.935 \\
\hline$>17$ & 0.77 & $(0.75-0.79)$ & $<0.001$ & 0.76 & $(0.71-0.80)$ & $<0.001$ & 0.78 & $(0.74-0.83)$ & $<0.001$ & 0.79 & $(0.75-0.83)$ & $<0.001$ & 0.84 & $(0.79-0.90)$ & $<0.001$ & 0.157 \\
\hline
\end{tabular}


Table 2. Cont.

\begin{tabular}{|c|c|c|c|c|c|c|c|c|c|c|c|c|c|c|c|c|}
\hline \multirow{2}{*}{ Risk Factor } & \multicolumn{3}{|c|}{ Total } & \multicolumn{3}{|c|}{ BI-RADS 1} & \multicolumn{3}{|c|}{ BI-RADS 2} & \multicolumn{3}{|c|}{ BI-RADS 3} & \multicolumn{3}{|c|}{ BI-RADS 4} & \multirow{2}{*}{ P-heterogeneity } \\
\hline & HR * & $95 \% \mathrm{CI}$ & $\mathbf{P}$ & HR * & $95 \% \mathrm{CI}$ & $p$-value & HR * & $95 \% \mathrm{CI}$ & $\mathbf{P}$ & $\mathrm{HR}^{*}$ & $95 \% \mathrm{CI}$ & $\mathbf{P}$ & HR * & $95 \% \mathrm{CI}$ & $\mathbf{P}$ & \\
\hline \multicolumn{17}{|c|}{ Menopausal Status } \\
\hline Premenopause & 1 & & & 1 & & & 1 & & & 1 & & & 1 & & & \\
\hline Postmenopause & 0.82 & $(0.78-0.85)$ & $<0.001$ & 0.87 & $(0.78-0.96)$ & 0.004 & 0.91 & $(0.84-0.98)$ & 0.011 & 0.82 & $(0.76-0.88)$ & $<0.001$ & 0.76 & $(0.68-0.85)$ & $<0.001$ & 0.051 \\
\hline \multicolumn{17}{|c|}{ Age at Menopause Among Menopaused Women } \\
\hline$\leq 49$ & 1 & & & 1 & & & 1 & & & 1 & & & 1 & & & \\
\hline$\leq 51$ & 1.18 & $(1.14-1.22)$ & $<0.001$ & 1.17 & $(1.09-1.25)$ & $<0.001$ & 1.09 & $(1.02-1.16)$ & 0.009 & 1.18 & $(1.10-1.26)$ & $<0.001$ & 1.10 & $(0.98-1.23)$ & 0.102 & 0.285 \\
\hline$>51$ & 1.42 & $(1.38-1.47)$ & $<0.001$ & 1.46 & $(1.37-1.56)$ & $<0.001$ & 1.28 & $(1.21-1.35)$ & $<0.001$ & 1.33 & $(1.25-1.41)$ & $<0.001$ & 1.24 & $(1.12-1.38)$ & $<0.001$ & 0.009 \\
\hline \multicolumn{17}{|c|}{ Hormone Replacement Therapy } \\
\hline No Usage & 0.81 & $(0.78-0.84)$ & $<0.001$ & 0.83 & $(0.77-0.9)$ & $<0.001$ & 0.9 & $(0.85-0.96)$ & 0.002 & 0.84 & $(0.79-0.89)$ & $<0.001$ & 0.88 & $(0.79-0.98)$ & 0.018 & 0.296 \\
\hline $\begin{array}{l}<1 \text { Year } \\
\geq 1 \text { Year }\end{array}$ & $\begin{array}{c}1 \\
1.18\end{array}$ & $(1.12-1.24)$ & $<0.001$ & $\begin{array}{c}1 \\
1.07\end{array}$ & $(0.94-1.21)$ & 0.312 & $\begin{array}{c}1 \\
1.13\end{array}$ & $(1.03-1.25)$ & 0.012 & $\begin{array}{c}1 \\
1.23\end{array}$ & $(1.12-1.34)$ & $<0.001$ & 1.34 & $(1.15-1.57)$ & 0.001 & 0.091 \\
\hline \multicolumn{17}{|c|}{ Oral Contraceptive Use } \\
\hline No Usage & 0.99 & $(0.97-1.02)$ & 0.617 & 0.97 & $(0.89-1.04)$ & 0.347 & 0.98 & $(0.93-1.04)$ & 0.582 & 1.00 & $(0.96-1.05)$ & 0.897 & 0.99 & $(0.93-1.05)$ & 0.786 & 0.902 \\
\hline$<6$ Months & 1 & & & 1 & & & 1 & & & 1 & & & 1 & & & \\
\hline$\geq 6$ Months & 0.97 & $(0.94-1.01)$ & 0.189 & 0.98 & $(0.89-1.08)$ & 0.634 & 0.98 & $(0.91-1.06)$ & 0.620 & 0.99 & $(0.93-1.05)$ & 0.674 & 0.98 & $(0.90-1.07)$ & 0.629 & 0.997 \\
\hline \multicolumn{17}{|c|}{ Family History (in First Degree Relative) } \\
\hline No & 1 & & & 1 & & & 1 & & & 1 & & & 1 & & & \\
\hline Yes & 2.00 & $(1.89-2.08)$ & $<0.001$ & 2.13 & $(1.85-2.44)$ & $<0.001$ & 1.82 & $(1.64-2.00)$ & $<0.001$ & 1.89 & $(1.75-2.04)$ & $<0.001$ & 1.96 & $(1.82-2.17)$ & $<0.001$ & 0.295 \\
\hline \multicolumn{17}{|l|}{ BMI $\left(\mathrm{Kg} / \mathrm{m}^{2}\right)$} \\
\hline$<18.5$ & 1 & & & 1 & & & 1 & & & 1 & & & 1 & & & \\
\hline$<23$ & 1.13 & $(1.06-1.19)$ & $<0.001$ & 1.23 & $(0.97-1.56)$ & 0.088 & 1.28 & $(1.08-1.53)$ & 0.005 & 1.14 & $(1.03-1.26)$ & 0.013 & 1.16 & $(1.07-1.27)$ & 0.001 & 0.685 \\
\hline$<25$ & 1.14 & $(1.07-1.21)$ & $<0.001$ & 1.34 & (1.05-1.69) & 0.017 & 1.38 & $(1.15-1.64)$ & 0.001 & 1.21 & (1.09-1.34) & 0.001 & 1.19 & (1.09-1.31) & 0.001 & 0.439 \\
\hline$>25$ & 1.23 & $(1.16-1.30)$ & $<0.001$ & 1.62 & $(1.28-2.05)$ & $<0.001$ & 1.6 & $(1.34-1.91)$ & $<0.001$ & 1.31 & $(1.18-1.45)$ & $<0.001$ & 1.28 & $(1.17-1.41)$ & $<0.001$ & 0.060 \\
\hline \multicolumn{17}{|c|}{ Smoking in Lifetime } \\
\hline Never Smoked & 1 & & & 1 & & & 1 & & & 1 & & & 1 & & & \\
\hline Ever Smoked & 1.01 & $(0.98-1.06)$ & 0.471 & 1.05 & $(0.94-1.17)$ & 0.422 & 0.98 & $(0.9-1.07)$ & 0.622 & 1.09 & $(1.02-1.16)$ & 0.009 & 1.00 & $(0.92-1.08)$ & 0.937 & 0.188 \\
\hline \multicolumn{17}{|c|}{ MeanDaysof Drinking per Weekduring the Last 1 Year } \\
\hline No Drinking & 1 & & & 1 & & & 1 & & & 1 & & & 1 & & & \\
\hline$\geq 1$ Day & 0.99 & $(0.96-1.01)$ & 0.181 & 0.95 & $(0.89-1.02)$ & 0.145 & 0.97 & $(0.93-1.02)$ & 0.272 & 0.99 & $(0.95-1.02)$ & 0.410 & 1.00 & $(0.96-1.04)$ & 0.986 & 0.583 \\
\hline \multicolumn{17}{|c|}{ MeanDaysof Physical Activity per Week } \\
\hline None & 0.96 & $(0.94-0.98)$ & $<0.001$ & 0.96 & $(0.90-1.02)$ & 0.171 & 0.98 & $(0.94-1.02)$ & 0.357 & 0.97 & $(0.94-1.00)$ & 0.087 & 0.97 & $(0.93-1.02)$ & 0.204 & 0.955 \\
\hline 1-3 Days/Week & 1 & & & 1 & & & 1 & & & 1 & & & 1 & & & \\
\hline$\geq 4$ Days/Week & 1.00 & $(0.97-1.02)$ & 0.675 & 1.01 & $(0.94-1.08)$ & 0.771 & 0.99 & $(0.94-1.05)$ & 0.822 & 1.00 & $(0.96-1.04)$ & 0.787 & 0.97 & $(0.92-1.02)$ & 0.183 & 0.767 \\
\hline
\end{tabular}

HR: hazard ratio; CI: confidence interval; ${ }^{*}$ Adjusted for listed variables in addition to age. 
Table 3. Association between known risk factors of breast cancer and breast cancer development by breast density in premenopausal women.

\begin{tabular}{|c|c|c|c|c|c|c|c|c|c|c|c|c|c|c|c|c|}
\hline \multirow{2}{*}{ Risk Factor } & \multicolumn{3}{|c|}{ Total } & \multicolumn{3}{|c|}{ BI-RADS 1} & \multicolumn{3}{|c|}{ BI-RADS 2} & \multicolumn{3}{|c|}{ BI-RADS 3} & \multicolumn{3}{|c|}{ BI-RADS 4} & \multirow{2}{*}{ P-heterogeneity } \\
\hline & HR * & $95 \% \mathrm{CI}$ & $\mathbf{P}$ & HR * & $95 \%$ CI & $p$-value & HR * & $95 \% \mathrm{CI}$ & $\mathbf{P}$ & $\mathrm{HR}^{*}$ & $95 \%$ CI & $\mathbf{P}$ & $\mathrm{HR}^{*}$ & $95 \% \mathrm{CI}$ & $\mathbf{P}$ & \\
\hline \multicolumn{17}{|l|}{ Age at Menarche } \\
\hline$\leq 15$ & 1 & & & 1 & & & 1 & & & 1 & & & 1 & & & \\
\hline $16-17$ & 0.89 & $(0.87-0.92)$ & $<0.001$ & 0.93 & $(0.84-1.02)$ & 0.112 & 0.89 & $(0.84-0.95)$ & 0.001 & 0.88 & $(0.85-0.92)$ & $<0.001$ & 0.91 & $(0.87-0.95)$ & $<0.001$ & 0.597 \\
\hline$>17$ & 0.78 & $(0.74-0.81)$ & $<0.001$ & 0.71 & $(0.62-0.83)$ & $<0.001$ & 0.75 & $(0.68-0.83)$ & $<0.001$ & 0.82 & $(0.76-0.87)$ & $<0.001$ & 0.83 & $(0.76-0.90)$ & $<0.001$ & 0.144 \\
\hline \multicolumn{17}{|c|}{ Oral Contraceptive Use } \\
\hline No Usage & 1.02 & $(0.98-1.06)$ & 0.381 & 1.09 & $(0.94-1.26)$ & 0.261 & 1.05 & $(0.96-1.16)$ & 0.302 & 1.00 & $(0.95-1.06)$ & 0.926 & 0.99 & $(0.92-1.06)$ & 0.716 & 0.548 \\
\hline$<6$ Months & 1 & & & 1 & & & 1 & & & 1 & & & 1 & & & \\
\hline$\geq 6$ Months & 1.00 & $(0.95-1.06)$ & 0.977 & 0.95 & $(0.78-1.17)$ & 0.649 & 1.06 & $(0.93-1.21)$ & 0.396 & 1.03 & $(0.95-1.12)$ & 0.527 & 0.99 & $(0.90-1.09)$ & 0.776 & 0.751 \\
\hline \multicolumn{17}{|c|}{ Family History (in First Degree Relative) } \\
\hline No & 1 & & & 1 & & & 1 & & & 1 & & & 1 & & & \\
\hline Yes & 2.04 & $(1.92-2.17)$ & $<0.001$ & 2.13 & $(1.67-2.70)$ & $<0.001$ & 1.92 & $(1.64-2.27)$ & $<0.001$ & 2.04 & $(1.85-2.22)$ & $<0.001$ & 1.92 & $(1.75-2.13)$ & $<0.001$ & 0.745 \\
\hline \multicolumn{17}{|l|}{ BMI $\left(\mathrm{Kg} / \mathrm{m}^{2}\right)$} \\
\hline$<18.5$ & 1 & & & 1 & & & 1 & & & 1 & & & 1 & & & \\
\hline$<23$ & 1.05 & $(0.98-1.12)$ & 0.173 & 0.93 & $(0.67-1.31)$ & 0.694 & 0.96 & $(0.76-1.22)$ & 0.735 & 1.08 & $(0.96-1.22)$ & 0.197 & 1.11 & $(1.01-1.22)$ & 0.031 & 0.564 \\
\hline$<25$ & 1.03 & $(0.96-1.11)$ & 0.351 & 0.95 & $(0.67-1.33)$ & 0.757 & 1.01 & $(0.80-1.28)$ & 0.936 & 1.10 & $(0.98-1.25)$ & 0.114 & 1.14 & $(1.03-1.26)$ & 0.011 & 0.641 \\
\hline$>25$ & 1.02 & $(0.95-1.09)$ & 0.631 & 0.86 & $(0.61-1.21)$ & 0.386 & 1.08 & $(0.85-1.37)$ & 0.518 & 1.15 & $(1.02-1.30)$ & 0.024 & 1.19 & $(1.08-1.32)$ & 0.001 & 0.323 \\
\hline \multicolumn{17}{|c|}{ Smoking in Lifetime } \\
\hline Never Smoked & 1 & & & 1 & & & 1 & & & 1 & & & 1 & & & \\
\hline Ever Smoked & 1.00 & $(0.96-1.06)$ & 0.886 & 0.86 & $(0.70-1.05)$ & 0.139 & 1.07 & $(0.95-1.21)$ & 0.242 & 1.04 & $(0.96-1.12)$ & 0.329 & 1.02 & $(0.93-1.11)$ & 0.742 & 0.319 \\
\hline \multicolumn{17}{|c|}{ MeanDaysof Drinking per Weekduring the Last 1 Year } \\
\hline No Drinking & 1 & & & 1 & & & 1 & & & 1 & & & 1 & & & \\
\hline$\geq 1$ Day & 1.00 & $(0.97-1.03)$ & 0.945 & 1.03 & $(0.93-1.14)$ & 0.540 & 0.99 & $(0.93-1.06)$ & 0.887 & 0.98 & $(0.94-1.02)$ & 0.456 & 1.01 & $(0.96-1.05)$ & 0.752 & 0.682 \\
\hline \multicolumn{17}{|c|}{ MeanDaysof Physical Activity per Week } \\
\hline None & 0.97 & $(0.94-1.00)$ & 0.02 & 0.97 & $(0.88-1.08)$ & 0.625 & 0.99 & $(0.93-1.07)$ & 0.834 & 0.98 & $(0.94-1.02)$ & 0.346 & 0.96 & $(0.92-1.01)$ & 0.126 & 0.880 \\
\hline 1-3 Days/Week & 1 & & & 1 & & & 1 & & & 1 & & & 1 & & & \\
\hline$\geq 4$ Days/Week & 0.98 & $(0.95-1.01)$ & 0.131 & 1.00 & $(0.88-1.13)$ & 0.967 & 1.03 & $(0.95-1.12)$ & 0.484 & 0.98 & $(0.93-1.03)$ & 0.348 & 0.94 & $(0.89-1.00)$ & 0.044 & 0.332 \\
\hline
\end{tabular}

HR: hazard ratio; CI: confidence interval; ${ }^{*}$ Adjusted for listed variables in addition to age. 
Table 4. Association between known risk factors of breast cancer and breast cancer development by breast density in postmenopausal women.

\begin{tabular}{|c|c|c|c|c|c|c|c|c|c|c|c|c|c|c|c|c|}
\hline \multirow{2}{*}{ Risk Factors } & \multicolumn{3}{|c|}{ Total } & \multicolumn{3}{|c|}{ BI-RADS 1} & \multicolumn{3}{|c|}{ BI-RADS 2} & \multicolumn{3}{|c|}{ BI-RADS 3} & \multicolumn{3}{|c|}{ BI-RADS 4} & \multirow{2}{*}{ P-heterogeneity } \\
\hline & HR * & $95 \% \mathrm{CI}$ & $\mathbf{P}$ & HR * & $95 \%$ CI & $p$-value & $\mathrm{HR}^{*}$ & $95 \% \mathrm{CI}$ & $\mathbf{P}$ & $\mathrm{HR}^{*}$ & $95 \%$ CI & $\mathbf{P}$ & $\mathrm{HR}^{*}$ & $95 \% \mathrm{CI}$ & $\mathbf{P}$ & \\
\hline \multicolumn{17}{|l|}{ Age at Menarche } \\
\hline$\leq 15$ & 1 & & & 1 & & & 1 & & & 1 & & & 1 & & & \\
\hline $16-17$ & 0.86 & $(0.83-0.88)$ & $<0.001$ & 0.86 & $(0.81-0.91)$ & $<0.001$ & 0.88 & $(0.84-0.93)$ & $<0.001$ & 0.88 & $(0.84-0.93)$ & $<0.001$ & 0.86 & $(0.78-0.94)$ & 0.001 & 0.909 \\
\hline$>17$ & 0.75 & $(0.73-0.78)$ & $<0.001$ & 0.76 & $(0.71-0.81)$ & $<0.001$ & 0.79 & $(0.75-0.84)$ & $<0.001$ & 0.76 & $(0.71-0.81)$ & $<0.001$ & 0.85 & $(0.75-0.95)$ & 0.006 & 0.333 \\
\hline \multicolumn{17}{|c|}{ Oral Contraceptive Use } \\
\hline No Usage & 0.94 & $(0.90-0.98)$ & 0.003 & 0.90 & $(0.83-0.99)$ & 0.024 & 0.94 & $(0.87-1.01)$ & 0.081 & 0.98 & $(0.90-1.06)$ & 0.579 & 0.97 & $(0.85-1.12)$ & 0.722 & 0.553 \\
\hline$<6$ Months & 1 & & & 1 & & & 1 & & & 1 & & & 1 & & & \\
\hline$\geq 6$ Months & 0.99 & $(0.94-1.05)$ & 0.726 & 1.01 & $(0.90-1.12)$ & 0.932 & 0.97 & $(0.88-1.07)$ & 0.545 & 0.98 & $(0.89-1.09)$ & 0.737 & 1.03 & $(0.85-1.24)$ & 0.792 & 0.918 \\
\hline \multicolumn{17}{|c|}{ Family History (in First Degree Relative) } \\
\hline No & 1 & & & 1 & & & 1 & & & 1 & & & 1 & & & \\
\hline Yes & 1.89 & $(1.75-2.04)$ & $<0.001$ & 2.08 & $(1.75-2.50)$ & $<0.001$ & 1.72 & $(1.52-2.00)$ & $<0.001$ & 1.59 & $(1.39-1.82)$ & $<0.001$ & 2.22 & $(1.82-2.70)$ & $<0.001$ & 0.014 \\
\hline \multicolumn{17}{|l|}{ BMI $\left(\mathrm{Kg} / \mathrm{m}^{2}\right)$} \\
\hline$<18.5$ & 1 & & & 1 & & & 1 & & & 1 & & & 1 & & & \\
\hline$<23$ & 1.35 & $(1.20-1.52)$ & $<0.001$ & 1.56 & $(1.11-2.19)$ & 0.011 & 1.77 & $(1.35-2.33)$ & $<0.001$ & 1.26 & $(1.04-1.52)$ & 0.017 & 1.48 & $(1.17-1.88)$ & 0.001 & 0.225 \\
\hline$<25$ & 1.44 & $(1.27-1.62)$ & $<0.001$ & 1.77 & $(1.26-2.49)$ & 0.001 & 1.94 & $(1.48-2.55)$ & $<0.001$ & 1.45 & $(1.20-1.76)$ & 0 & 1.53 & $(1.19-1.96)$ & 0.001 & 0.333 \\
\hline$>25$ & 1.67 & $(1.48-1.88)$ & $<0.001$ & 2.38 & $(1.70-3.34)$ & $<0.001$ & 2.36 & $(1.80-3.09)$ & $<0.001$ & 1.64 & $(1.35-1.98)$ & $<0.001$ & 1.79 & $(1.40-2.29)$ & $<0.001$ & 0.008 \\
\hline \multicolumn{17}{|c|}{ Smoking in Lifetime } \\
\hline Never Smoked & 1 & & & 1 & & & 1 & & & 1 & & & 1 & & & \\
\hline Ever Smoked & 1.04 & $(0.97-1.11)$ & 0.276 & 1.14 & $(0.99-1.31)$ & 0.065 & 0.89 & $(0.79-1.01)$ & 0.079 & 1.20 & $(1.08-1.34)$ & 0.001 & 0.91 & $(0.74-1.11)$ & 0.347 & 0.001 \\
\hline \multicolumn{17}{|c|}{ MeanDaysof Drinking per Weekduring the Last 1 Year } \\
\hline No Drinking & 1 & & & 1 & & & 1 & & & 1 & & & 1 & & & \\
\hline$\geq 1$ Day & 1.04 & $(1.00-1.08)$ & 0.062 & 1.10 & $(1.01-1.20)$ & 0.027 & 1.05 & $(0.98-1.12)$ & 0.175 & 1.00 & $(0.94-1.07)$ & 0.900 & 1.04 & $(0.93-1.16)$ & 0.509 & 0.374 \\
\hline \multicolumn{17}{|c|}{ MeanDaysof Physical Activity per Week } \\
\hline None & 0.94 & $(0.91-0.97)$ & 0.001 & 0.95 & $(0.88-1.02)$ & 0.169 & 0.96 & $(0.90-1.02)$ & 0.156 & 0.96 & $(0.90-1.02)$ & 0.136 & 1.03 & $(0.93-1.15)$ & 0.534 & 0.643 \\
\hline 1-3 Days/Week & 1 & & & 1 & & & 1 & & & 1 & & & 1 & & & \\
\hline$\geq 4$ Days/Week & 1.02 & $(0.98-1.06)$ & 0.380 & 1.03 & $(0.94-1.12)$ & 0.537 & 0.96 & $(0.90-1.03)$ & 0.297 & 1.03 & $(0.97-1.11)$ & 0.349 & 1.08 & $(0.96-1.21)$ & 0.220 & 0.264 \\
\hline
\end{tabular}

HR: hazard ratio; CI: confidence interval; * Adjusted for listed variables in addition to age, age at menopause, and hormone replacement therapy use. 
In postmenopausal participants, in addition to age at menopause ( $>51$ years old, shown in Table 2), a family history of breast cancer, BMI $\geq 25 \mathrm{~kg} / \mathrm{m}^{2}$, and smoking history showed a heterogeneous association with BI-RADS density categories (Table 4). Those with family history of breast cancer showed an increased risk of breast cancer with a HR of 2.08 (95\% CI $=1.75-2.50), 1.72(95 \% \mathrm{CI}=$ $1.52-2.00), 1.59(95 \% \mathrm{CI}=1.39-1.82), 2.22(95 \% \mathrm{CI}=1.82-2.70)$ in BI-RADS category of $1,2,3$, and 4 , respectively (P-heterogeneity $=0.014$ ). Compared with BMI $<18.5 \mathrm{~kg} / \mathrm{m}^{2}$, those with BMI $\geq 25 \mathrm{~kg} / \mathrm{m}^{2}$ had an HR of 2.38 (95\% CI = 1.70-3.34), $2.36(95 \% \mathrm{CI}=1.80-3.09), 1.64(95 \% \mathrm{CI}=1.35-1.98)$, and 1.79 $(95 \% \mathrm{CI}=1.40-2.29)$ for those in BI-RADS categories $1,2,3$, and 4, respectively (P-heterogeneity $=$ 0.008). Smoking increased the risk of breast cancer significantly in participants with BI-RADS density category $3(\mathrm{HR}=1.20 ; 95 \% \mathrm{CI}=1.08-1.34)$; however, smoking did not show an association in other categories (P-heterogeneity $=0.001)$.

\section{Discussion}

To the best of our knowledge, this is the one of the largest population-based studies that has investigated the heterogeneity of the effect of risk factors on breast cancer development across BI-RADS density categories. Age and age at menopause ( $>51$ years vs. $\leq 49$ years) showed a heterogeneous association with breast cancer across density categories. Among the participants who underwent menopause, a family history of breast cancer, BMI $\geq 25 \mathrm{~kg} / \mathrm{m}^{2}$, and smoking showed a heterogeneous effect on breast cancer across the BI-RADS density categories. In premenopausal participants, risk factors did not show a heterogeneous association with breast cancer across the BI-RADS density categories.

In the Korean population, age-specific breast cancer incidence increases up to the age of 45-49 years, and then decreases with further increases in age [16]. In the Korean population, age-specific breast cancer incidence increases up to the age of 45-49 years, and then decreases with further increases in age [16]. A higher incidence rate of breast cancer in women aged $<50$ years is a distinct pattern in Asian countries where approximately $50 \%$ of breast cancers are diagnosed among women under 50 [2]. Considering that the cumulative proportion of women who experience menopause before 50 and 55 years of age is $46.0 \%$ and $90.3 \%$ [17], premenopausal breast cancer development is more common than post-menopausal breast cancer. Not only among Korean women but also among other Asian women, breast cancer incidence plateaus or decreases above the age of 50 , which is in contrast to the continuous increase in age-specific breast cancer incidence rates with increased age among Western women [18]. In this study, $>80 \%$ of the participants with BI-RADS density category 1 were aged $\geq 50$ years; $70 \%$ of the participants with BI-RADS density category 4 were aged 40-49. Considering the age-related decline in breast density [19], the association of age with breast density may reflect the age-specific breast cancer incidence rate in Korea. The result is in line with a previous study that showed that high breast density was related to the aggressiveness of breast tumors, especially in younger women [20].

A previous study suggested that menopause is more strongly associated with breast density than age [21]. Several studies have investigated the association between mammographic density and breast cancer based on menopausal status and hormone replacement therapy. A study suggested that despite the absence of statistical significance, the strength of the association between density and breast cancer was different, with the strongest association in premenopausal women and postmenopausal women using hormone replacement therapy [8,22-24]. However, another study showed the opposite result; the effect of mammographic density was not modified by menopausal status or use of hormone replacement therapy [13]. Reciprocity of the interaction means that if $A$ is an effect modifier of $B$, then B modifies the effect of A [25]; therefore, when the effect of breast density is significantly different based on other factors, we can expect that the associations of the factor would be different based on the breast density. Kerlikowske et al. showed that women with BI-RADS density category 3 and 4 using hormone replacement therapy had a higher risk of breast cancer than those who did not; this association was not observed in women with BI-RADS density category 1 and 2 [23], similar to our result. However, our results did not show a significant heterogeneity. Despite the lower proportion 
of women who had ever used hormone replacement therapy than that in a previous study in the United States [21], the heterogenous effects were comparable. More advanced age at menopause was associated with dense breasts [6] and an increased risk of breast cancer [26]; however, the association of age at menopause and breast cancer based on breast density has rarely been studied. In Korean women, the current mean age at menopause is lower than that in other countries such as the United Kingdom, Australia, or Japan but shows an increasing trend [17], which may possibly contribute to the increased breast cancer-related burden. In this study, despite a similar association, advanced age at menopause ( $>51$ years) was associated more with breast cancer in women with low breast density than in women with dense breast tissue.

$\mathrm{BMI}$ is a well-known risk factor for the development of breast cancer in postmenopausal women because of the aromatization of androgens into estrogens, but not premenopausal breast cancer where increased BMI may have a protective effect [27]. However, in this study, even premenopausal women with dense breasts (BI-RADS density category 3 and 4 ) had a slightly increased risk of breast cancer with increased BMI. The combined effect of breast density and BMI on breast cancer has been studied well. The suggested mechanism is that obesity-related insulin deregulation and the adipokine-associated inflammatory response may activate proliferation [28]. In this study, in postmenopausal women with BI-RADS density category 1 , BMI had a more prominent effect on breast cancer; this effect decreased as the BI-RADS density category increased. If those with BI-RADS density category 4 had a higher baseline risk of breast cancer, then the added effect of BMI might be lower. Previous studies on the relationship of the effect of breast density and BMI showed inconsistent results; more evident interactions were observed in postmenopausal women [29] or premenopausal women due to a higher risk of estrogen receptor-negative cancer [30]. However, other studies did not find a relationship between breast density and BMI in breast cancer [31], irrespective of menopausal status [10]. In both developed and developing countries, the prevalence of obesity is increasing, especially in younger age groups in developing countries [32]. However, in Korea, prevalence of obesity, defined as BMI $\geq 25 \mathrm{~kg} / \mathrm{m}^{2}$, has not increased in women. Especially in women aged 20-39 and 40-59 years old, the prevalence of obesity has decreased [33], which is different from the trend in other countries [32]. The unique pattern of changes in BMI in Korea might contribute to the differing results.

Several studies have shown that the association between breast density and the risk of breast cancer is stronger in women with a family history of breast cancer than those without a family history $[9,11,34]$. The heritability of mammographic density within family members has been shown before $[35,36]$. A recent study suggested that genetic markers in mammographic density such as percent density and dense area show a shared genetic origin and biological pathways with breast cancer [37]. In this study, we observed the heterogeneous effect of a family history of breast cancer based on BI-RADS density category in postmenopausal women. In women with BI-RADS density category 4 , those with a family history of breast cancer showed an increased breast cancer risk; however, in women with BI-RADS density category 3 , the increment was less. Considering that the direction and strength of associations were comparable in all BI-RADS density categories, the significant P-heterogeneity may be due to the large sample size of the study subjects.

Despite the well-known carcinogenic effect of smoking, there has been a debate on the association between smoking and breast cancer. However, recent studies demonstrated that smoking increased the risk of breast cancer [38], as well as postmenopausal breast cancer risk [39]. In this study, we did not observe an overall increased risk of smoking in all, premenopausal, or postmenopausal women. It could be attributed to the low prevalence of ever-smoking in Korean women in this study's subjects, which was similar to that reported in previous studies $[40,41]$. However, in postmenopausal women, a heterogeneous effect of smoking was observed; smoking significantly increased the risk in women with BI-RADS density category 3 and marginally decreased the risk in women with BI-RADS density category 2. Smoking, due to its antiestrogenic effect on breast tissue, is associated with a decrease in breast density $[42,43]$. Further investigation is required to understand the effects of smoking on breast density and its association with the risk of breast cancer. 
The effect of the decrease in risk with increased age at menarche and a non-significant association of oral contraceptive use irrespective of the breast density category were comparable with findings in previous studies [26,44]. Lower use of oral contraceptives in the Korean population may be the cause of the non-significant association [45]. Light drinking is not associated with most types of cancer and only leads to a mild increases in the risk of breast cancer [46]. The non-significant association of alcohol consumption might be attributed to the low alcohol consumption among Korean women [47]. In Korean women, the prevalence of drinking, defined as $\geq 1$ drink of alcohol per month during the last year, was around 40\% [41] and the average alcohol consumption was $8 \mathrm{~g} /$ day [48].

There are several limitations of this study. First, the study population comprised cancer screening examinees and their baseline characteristics may not be comparable with those of non-examinees. Considering that breast cancer screening participation rate in 2009-2010 was approximately 45\% [49] and we included all breast cancer examinees, our study covered almost half of the target population. However, considering that women with lower levels of household income or education participate less in breast cancer screening programs [50], a possible selection bias still remains. Secondly, the BI-RADS density classification provides information to physicians regarding the likelihood of missing a lesion that may be masked by dense tissues; BI-RADS does not quantify breast cancer risk exactly [51]. The BI-RADS density categories were reported by radiologists at many different screening units. However, the inter-observer agreement on BI-RADS categories is substantial [52,53] and a mammographic education program has been conducted to standardize the performance of radiologists in Korea [54]. Furthermore, although the automated breast density and BI-RADS categories showed modest agreement, their association with breast cancer was similar [55], suggesting that the HRs in this study are robust. Thirdly, the information regarding all risk factors except BMI were obtained from self-administered questionnaires; thus, there may be a bias in the responses. However, this would lead to non-differential misclassification and the effects on the results would not be significant [56]. Regarding risk factors, due to a lack of information, some important risk factors for breast cancer, such as parity-related factors, could not be considered. Therefore, the residual confounding effect of unaccounted variables or variables with broad categories may affect the results. In addition, several risk factors, such as BMI, oral contraceptive use, or hormone replacement therapy use, can be changed during one's lifetime; however, we considered these risk factors at a single time point at their screening during the year of 2009-2010. Fourthly, cancer incidence was identified using the ICD-10 code and catastrophic illness registry in the National Health Insurance Service (NHIS) database, which has the potential for misclassification. However, using the catastrophic illness registry is related to the reimbursement of co-payment, requiring relevant clinical information by the insurance administration. In addition, it covers more than $97 \%$ of cancer patients in the Korea Central Cancer Registry. Thus, bias regarding the definition of breast cancer incidence would be minimal. When we separated invasive breast cancer and ductal carcinoma in situ, the results were comparable with the original results, supporting the robustness of the results. The follow-up time of $\geq 8$ years would not be enough to identify all breast cancers. Breast cancer risk assessment estimates mostly 5-year risk but long-term risk assessment for 10 years or more is also needed [57]. Thus, the follow-up period of this study represents an intermediate time point.

Despite these limitations, our study has a number of strengths including the large sample size and prospective design. The follow-up period was $>8$ years and enabled us to study the shortand intermediate-term effects of risk factors and breast density on breast cancer. In addition, the study population covers a large proportion of the female population in Korea and various risk factors interacting with breast density were considered together. 


\section{Materials and Methods}

\subsection{Study Population}

The NHIS in Korea supports biennial health examinations for individuals aged $\geq 40$ years. It also supports the screening of stomach, liver, colon, breast, and cervical cancer in the eligible population. Participants of health examinations and cancer screening are asked to complete self-reported questionnaires, which collect information on lifestyle factors, family history of chronic diseases and cancer, and reproductive factors. The information in the questionnaires and results of health examinations and cancer screening are collected through the NHIS screening database. All participants provided consent that allowed the transfer of information to the NHIS database.

For breast cancer, NHIS supports biennial mammographic screening for women aged $\geq 40$ years. Approximately $40 \%$ of women who are eligible participate in the screening; approximately 3 million mammographic screenings are performed each year. During screening, data on the BI-RADS density assessment and BI-RADS assessment categories were collected. Breast cancer incidence was defined via linking the database with the NHIS medical usage database to obtain the information related to either invasive breast cancer (C50) or ductal carcinoma in situ (D05) and catastrophic illness codes up to December 2018. Upon reviewing the study proposal and request to the National Health Insurance Sharing Service, the NHIS database was made available for research. The study proposal was approved by the Institutional Review Board of the Hanyang University College of Medicine (IRB no. HYI-18-175-1).

We identified 5,317,312 women who participated in health examinations and breast cancer screenings between 2009 and 2010. Among these, women aged $\geq 75$ and women who had a medical usage record for any type of cancer with catastrophic illnesses code before the date of breast cancer screening or within 3 months from the date of breast cancer screening $(n=278,461)$ were excluded. The remaining 5,038,851 were included in the analysis as subjects. Women who newly acquired medical usage record for breast cancer (C50 and D05) with a catastrophic illness code $(n=55,538)$ by December 2018 were defined as incident breast cancer cases. In addition, 141,345 women with missing information on breast density or with breast implants were excluded; the remaining 4,897,506 women including 54,164 breast cancer cases were included in the analysis for the analysis stratified by BI-RADS density categories.

\subsection{Mammographic Density}

BI-RADS breast density was classified into four categories: 1, almost entirely fat; 2, scattered fibroglandular densities; 3, heterogeneously dense; and 4, extremely dense.

\subsection{Risk Factors for Breast Cancer}

Information on breast cancer risk factors was obtained from self-reported questionnaires. The questionnaire for health examination included smoking history in one's lifetime, mean days of drinking per week during the last 1 year, and mean days of physical activity during the last one week. BMI was calculated based on the weight and height measured during the health examination. Reproductive factors, including age at menarche, menopausal status, age at menopause, use of hormone replacement therapy, and oral contraceptive, and family history of cancer in first-degree relatives were obtained from the questionnaire for cancer screening.

\subsection{Statistical Analysis}

The distribution of breast cancer risk factors as a whole and according to the BI-RADS density categories was presented as numbers and percentages. The effect of each risk factor on breast cancer was analyzed using the Cox proportional-hazard regression model adjusted for other risk factors. The results are presented as hazard ratio (HR) and 95\% confidence intervals (CIs) for the total number of participants and stratified by BI-RADS density categories. The follow-up was considered from the 
date of breast cancer screening during the year of 2009-2010 up to December 31, 2018, date of death, or date of any cancer incidence (based on which date came first). Incident breast cancer was considered as an event; death, incidence of other types of cancer, and no incidence of cancer were censored. Additionally, the effect of each risk factor on breast cancer and the stratification by BI-RADS breast density categories were evaluated according to the menopausal status. Heterogeneity of the effect of each risk factor across the BI-RADS density categories was assessed using $\mathrm{I}^{2}$ statistics. Analyses were conducted using SAS 9.4 (SAS Institute, Cary, NC, USA) and R software (version 3.5.0) during March-December 2019.

\section{Conclusions}

In summary, some known risk factors of breast cancer showed a heterogeneous effect on breast cancer across breast density categories, especially in postmenopausal women. The association of genetic risk factors or family history, known breast cancer risk factors, especially reproductive factors with breast density is under investigation. Among various hypotheses regarding the mechanism of effect of breast density on breast cancer risk, this research focused on the interaction effect. However, studies have suggested the mediation effect of breast density on the association between known risk factors and breast cancer [58,59]. The role of breast density in breast cancer risk in Asian women, including its mediation effect, needs to be considered in future studies.

Author Contributions: Conceptualization, B.P.; Data curation, J.Y.; Formal analysis, S.-E.L. and Y.S.C.; Investigation, S.-E.L., H.A. and Y.S.C.; Project administration, H.A. and J.Y.; Supervision, B.P.; Writing-original draft, B.P. and S.-E.L.; Writing-review \& editing, B.P. All authors have read and agreed to the published version of the manuscript.

Funding: This research was funded by National Research Foundation of Korea: NRF-2019R1H1A1079862. Korean Foundation for Cancer Research: 2019.

Acknowledgments: This work was supported by the Basic Science Research Program through the National Research Foundation of Korea (NRF) funded by the Ministry of Science, ICT and Future Planning (NRF-2019R1H1A1079862) and a research grant in 2019 by the Korean Foundation for Cancer Research, Seoul, Republic of Korea.

Conflicts of Interest: The authors declared no conflict of interest.

\section{References}

1. Bray, F.; Ferlay, J.; Soerjomataram, I.; Siegel, R.L.; Torre, L.A.; Jemal, A. Global cancer statistics 2018: GLOBOCAN estimates of incidence and mortality worldwide for 36 cancers in 185 countries. CA Cancer J. Clin. 2018, 68, 394-424. [CrossRef]

2. Youlden, D.R.; Cramb, S.M.; Yip, C.H.; Baade, P.D. Incidence and mortality of female breast cancer in the Asia-Pacific region. Cancer Biol. Med. 2014, 11, 101-115.

3. McCormack, V.A.; dos Santos Silva, I. Breast density and parenchymal patterns as markers of breast cancer risk: A meta-analysis. Cancer Epidemiol. Biomark. Prev. A Publ. Am. Assoc. Cancer Res. Cosponsored Am. Soc. Prev. Oncol. 2006, 15, 1159-1169. [CrossRef] [PubMed]

4. Shin, J.; Lee, J.E.; Ko, H.Y.; Nguyen, T.L.; Nam, S.J.; Hopper, J.L.; Song, Y.M. Association between mammographic density and tumor marker-defined breast cancer subtypes: A case-control study. Eur. J. Cancer Prev. Off. J. Eur. Cancer Prev. Organ. (ECP) 2018, 27, 239-247. [CrossRef] [PubMed]

5. Yaghjyan, L.; Colditz, G.A.; Rosner, B.; Bertrand, K.A.; Tamimi, R.M. Reproductive factors related to childbearing and mammographic breast density. Breast Cancer Res. Treat. 2016, 158, 351-359. [CrossRef] [PubMed]

6. Sung, H.; Ren, J.; Li, J.; Pfeiffer, R.M.; Wang, Y.; Guida, J.L.; Fang, Y.; Shi, J.; Zhang, K.; Li, N.; et al. Breast cancer risk factors and mammographic density among high-risk women in urban China. NPJ Breast Cancer 2018, 4, 3. [CrossRef] [PubMed]

7. Andersen, Z.J.; Baker, J.L.; Bihrmann, K.; Vejborg, I.; Sorensen, T.I.; Lynge, E. Birth weight, childhood body mass index, and height in relation to mammographic density and breast cancer: A register-based cohort study. Breast Cancer Res. 2014, 16, R4. [CrossRef] 
8. Yaghjyan, L.; Colditz, G.A.; Rosner, B.; Tamimi, R.M. Mammographic breast density and breast cancer risk: Interactions of percent density, absolute dense, and non-dense areas with breast cancer risk factors. Breast Cancer Res. Treat. 2015, 150, 181-189. [CrossRef]

9. Maskarinec, G.; Nakamura, K.L.; Woolcott, C.G.; Conroy, S.M.; Byrne, C.; Nagata, C.; Ursin, G.; Vachon, C.M. Mammographic density and breast cancer risk by family history in women of white and Asian ancestry. Cancer Causes Control 2015, 26, 621-626. [CrossRef]

10. Conroy, S.M.; Woolcott, C.G.; Koga, K.R.; Byrne, C.; Nagata, C.; Ursin, G.; Vachon, C.M.; Yaffe, M.J.; Pagano, I.; Maskarinec, G. Mammographic density and risk of breast cancer by adiposity: An analysis of four case-control studies. Int. J. Cancer 2012, 130, 1915-1924. [CrossRef]

11. Maskarinec, G.; Dartois, L.; Delaloge, S.; Hopper, J.; Clavel-Chapelon, F.; Baglietto, L. Tumor characteristics and family history in relation to mammographic density and breast cancer: The French E3N cohort. Cancer Epidemiol. 2017, 49, 156-160. [CrossRef] [PubMed]

12. Woolcott, C.G.; Koga, K.; Conroy, S.M.; Byrne, C.; Nagata, C.; Ursin, G.; Vachon, C.M.; Yaffe, M.J.; Pagano, I.; Maskarinec, G. Mammographic density, parity and age at first birth, and risk of breast cancer: An analysis of four case-control studies. Breast Cancer Res. Treat. 2012, 132, 1163-1171. [CrossRef] [PubMed]

13. Yaghjyan, L.; Tamimi, R.M.; Bertrand, K.A.; Scott, C.G.; Jensen, M.R.; Pankratz, V.S.; Brandt, K.; Visscher, D.; Norman, A.; Couch, F.; et al. Interaction of mammographic breast density with menopausal status and postmenopausal hormone use in relation to the risk of aggressive breast cancer subtypes. Breast Cancer Res. Treat. 2017, 165, 421-431. [CrossRef] [PubMed]

14. Szklo, M.; Nieto, F.J. Epidemiology: Beyond the Basics; Jones \& Bartlett Publishers: Burlington, MA, USA, 2014.

15. Yip, C.H.; Taib, N.A.; Song, C.V.; Pritam Singh, R.K.; Agarwal, G. Early Diagnosis of Breast Cancer in the Absence of Population-Based Mammographic Screening in Asia. Curr. Breast Cancer Rep. 2018, 10, 148-156. [CrossRef]

16. Jung, K.-W.; Won, Y.-J.; Kong, H.-J.; Lee, E.S. Cancer Statistics in Korea: Incidence, Mortality, Survival, and Prevalence in 2016. Cancer Res. Treat. 2019, 51, 417-430. [CrossRef]

17. Park, C.Y.; Lim, J.Y.; Park, H.Y. Age at natural menopause in Koreans: Secular trends and influences thereon. Menopause (New York N.Y.) 2018, 25, 423-429. [CrossRef]

18. Sung, H.; Rosenberg, P.S.; Chen, W.Q.; Hartman, M.; Lim, W.Y.; Chia, K.S.; Wai-Kong Mang, O.; Chiang, C.J.; Kang, D.; Ngan, R.K.; et al. Female breast cancer incidence among Asian and Western populations: More similar than expected. J. Natl. Cancer Inst. 2015, 107. [CrossRef]

19. Burton, A.; Maskarinec, G.; Perez-Gomez, B.; Vachon, C.; Miao, H.; Lajous, M.; Lopez-Ridaura, R.; Rice, M.; Pereira, A.; Garmendia, M.L.; et al. Mammographic density and ageing: A collaborative pooled analysis of cross-sectional data from 22 countries worldwide. PLoS Med. 2017, 14, e1002335. [CrossRef]

20. Bertrand, K.A.; Tamimi, R.M.; Scott, C.G.; Jensen, M.R.; Pankratz, V.; Visscher, D.; Norman, A.; Couch, F.; Shepherd, J.; Fan, B.; et al. Mammographic density and risk of breast cancer by age and tumor characteristics. Breast Cancer Res. 2013, 15, R104. [CrossRef]

21. Boyd, N.; Martin, L.; Stone, J.; Little, L.; Minkin, S.; Yaffe, M. A longitudinal study of the effects of menopause on mammographic features. Cancer Epidemiol. Biomark. Prev. A Publ. Am. Assoc. Cancer Res. Cosponsored Am. Soc. Prev. Oncol. 2002, 11, 1048-1053.

22. Yaghjyan, L.; Colditz, G.A.; Rosner, B.; Tamimi, R.M. Mammographic breast density and breast cancer risk by menopausal status, postmenopausal hormone use and a family history of breast cancer. Cancer Causes Control 2012, 23, 785-790. [CrossRef] [PubMed]

23. Kerlikowske, K.; Cook, A.J.; Buist, D.S.; Cummings, S.R.; Vachon, C.; Vacek, P.; Miglioretti, D.L. Breast cancer risk by breast density, menopause, and postmenopausal hormone therapy use. J. Clin. Oncol. Off. J. Am. Soc. Clin. Oncol. 2010, 28, 3830-3837. [CrossRef] [PubMed]

24. Hou, N.; Hong, S.; Wang, W.; Olopade, O.I.; Dignam, J.J.; Huo, D. Hormone replacement therapy and breast cancer: Heterogeneous risks by race, weight, and breast density. J. Natl. Cancer Inst. 2013, 105, 1365-1372. [CrossRef] [PubMed]

25. Szklo, M.; Nietro, F.J. Epidemiology: Beyond the Basic, 2nd ed.; Jones and Bartlett: Burlington, MA, USA, 2007.

26. Collaborative Group on Hormonal Factors in Breast Cancer. Menarche, menopause, and breast cancer risk: Individual participant meta-analysis, including 118964 women with breast cancer from 117 epidemiological studies. Lancet Oncol. 2012, 13, 1141-1151. [CrossRef] 
27. Chen, Y.; Liu, L.; Zhou, Q.; Imam, M.U.; Cai, J.; Wang, Y.; Qi, M.; Sun, P.; Ping, Z.; Fu, X. Body mass index had different effects on premenopausal and postmenopausal breast cancer risks: A dose-response meta-analysis with 3,318,796 subjects from 31 cohort studies. BMC Public Health 2017, 17, 936. [CrossRef] [PubMed]

28. Sanchez-Jimenez, F.; Perez-Perez, A.; de la Cruz-Merino, L.; Sanchez-Margalet, V. Obesity and Breast Cancer: Role of Leptin. Front. Oncol. 2019, 9, 596. [CrossRef]

29. Engmann, N.J.; Scott, C.G.; Jensen, M.R.; Winham, S.; Miglioretti, D.L.; Ma, L.; Brandt, K.; Mahmoudzadeh, A.; Whaley, D.H.; Hruska, C.; et al. Combined effect of volumetric breast density and body mass index on breast cancer risk. Breast Cancer Res. Treat. 2019, 177, 165-173. [CrossRef]

30. Shieh, Y.; Scott, C.G.; Jensen, M.R.; Norman, A.D.; Bertrand, K.A.; Pankratz, V.S.; Brandt, K.R.; Visscher, D.W.; Shepherd, J.A.; Tamimi, R.M.; et al. Body mass index, mammographic density, and breast cancer risk by estrogen receptor subtype. Breast Cancer Res. 2019, 21, 48. [CrossRef]

31. Harris, H.R.; Tamimi, R.M.; Willett, W.C.; Hankinson, S.E.; Michels, K.B. Body size across the life course, mammographic density, and risk of breast cancer. Am. J. Epidemiol. 2011, 174, 909-918. [CrossRef]

32. Ng, M.; Fleming, T.; Robinson, M.; Thomson, B.; Graetz, N.; Margono, C.; Mullany, E.C.; Biryukov, S.; Abbafati, C.; Abera, S.F.; et al. Global, regional, and national prevalence of overweight and obesity in children and adults during 1980-2013: A systematic analysis for the Global Burden of Disease Study 2013. Lancet (London, England) 2014, 384, 766-781. [CrossRef]

33. Kang, H.T.; Shim, J.Y.; Lee, H.R.; Park, B.J.; Linton, J.A.; Lee, Y.J. Trends in prevalence of overweight and obesity in Korean adults, 1998-2009: The Korean National Health and Nutrition Examination Survey. J. Epidemiol. 2014, 24, 109-116. [CrossRef] [PubMed]

34. Ahern, T.P.; Sprague, B.L.; Bissell, M.C.S.; Miglioretti, D.L.; Buist, D.S.M.; Braithwaite, D.; Kerlikowske, K. Family History of Breast Cancer, Breast Density, and Breast Cancer Risk in a U.S. Breast Cancer Screening Population. Cancer Epidemiol. Biomark. Prev. A Publ. Am. Assoc. Cancer Res. Cosponsored Am. Soc. Prev. Oncol. 2017, 26, 938-944. [CrossRef] [PubMed]

35. Brand, J.S.; Humphreys, K.; Thompson, D.J.; Li, J.; Eriksson, M.; Hall, P.; Czene, K. Volumetric mammographic density: Heritability and association with breast cancer susceptibility loci. J. Natl. Cancer Inst. 2014, 106. [CrossRef] [PubMed]

36. Varghese, J.S.; Smith, P.L.; Folkerd, E.; Brown, J.; Leyland, J.; Audley, T.; Warren, R.M.; Dowsett, M.; Easton, D.F.; Thompson, D.J. The heritability of mammographic breast density and circulating sex-hormone levels: Two independent breast cancer risk factors. Cancer Epidemiol. Biomark. Prev. A Publ. Am. Assoc. Cancer Res. Cosponsored Am. Soc. Prev. Oncol. 2012, 21, 2167-2175. [CrossRef]

37. Lindström, S.; Thompson, D.J.; Paterson, A.D.; Li, J.; Gierach, G.L.; Scott, C.; Stone, J.; Douglas, J.A.; dos-Santos-Silva, I.; Fernandez-Navarro, P. Genome-wide association study identifies multiple loci associated with both mammographic density and breast cancer risk. Nat. Commun. 2014, 5, 5303. [CrossRef]

38. Gaudet, M.M.; Gapstur, S.M.; Sun, J.; Diver, W.R.; Hannan, L.M.; Thun, M.J. Active smoking and breast cancer risk: Original cohort data and meta-analysis. J. Natl. Cancer Inst. 2013, 105, 515-525. [CrossRef]

39. Nyante, S.J.; Gierach, G.L.; Dallal, C.M.; Freedman, N.D.; Park, Y.; Danforth, K.N.; Hollenbeck, A.R.; Brinton, L.A. Cigarette smoking and postmenopausal breast cancer risk in a prospective cohort. Br. J. Cancer 2014, 110, 2339-2347. [CrossRef]

40. Park, S.; Jee, S.H.; Shin, H.R.; Park, E.H.; Shin, A.; Jung, K.W.; Hwang, S.S.; Cha, E.S.; Yun, Y.H.; Park, S.K.; et al. Attributable fraction of tobacco smoking on cancer using population-based nationwide cancer incidence and mortality data in Korea. BMC Cancer 2014, 14, 406. [CrossRef]

41. Web page of Korean National Health \& Nutrition Examination Survey. Available online: https://knhanes.cdc. go.kr/knhanes/eng/index.do (accessed on 9 May 2020).

42. Jacobsen, K.K.; Lynge, E.; Vejborg, I.; Tjonneland, A.; von Euler-Chelpin, M.; Andersen, Z.J. Cigarette smoking and mammographic density in the Danish Diet, Cancer and Health cohort. Cancer Causes Control 2016, 27, 271-280. [CrossRef]

43. Butler, L.M.; Gold, E.B.; Conroy, S.M.; Crandall, C.J.; Greendale, G.A.; Oestreicher, N.; Quesenberry, C.P., Jr.; Habel, L.A. Active, but not passive cigarette smoking was inversely associated with mammographic density. Cancer Causes Control 2010, 21, 301-311. [CrossRef]

44. Zhu, H.; Lei, X.; Feng, J.; Wang, Y. Oral contraceptive use and risk of breast cancer: A meta-analysis of prospective cohort studies. Eur. J. Contracept. Reprod. Health Care Off. J. Eur. Soc. Contracept. 2012, 17, 402-414. [CrossRef] [PubMed] 
45. Park, B.; Park, S.; Shin, H.R.; Shin, A.; Yeo, Y.; Choi, J.Y.; Jung, K.W.; Kim, B.G.; Kim, Y.M.; Noh, D.Y.; et al. Population attributable risks of modifiable reproductive factors for breast and ovarian cancers in Korea. $B M C$ Cancer 2016, 16, 5. [CrossRef] [PubMed]

46. Choi, Y.J.; Myung, S.K.; Lee, J.H. Light Alcohol Drinking and Risk of Cancer: A Meta-Analysis of Cohort Studies. Cancer Res. Treat. 2018, 50, 474-487. [CrossRef] [PubMed]

47. Ryu, S.Y.; Crespi, C.M.; Maxwell, A.E. Drinking patterns among Korean adults: Results of the 2009 Korean community health survey. J. Prev. Med. Public Health 2013, 46, 183-191. [CrossRef] [PubMed]

48. Park, S.; Shin, H.R.; Lee, B.; Shin, A.; Jung, K.W.; Lee, D.H.; Jee, S.H.; Cho, S.I.; Park, S.K.; Boniol, M.; et al. Attributable fraction of alcohol consumption on cancer using population-based nationwide cancer incidence and mortality data in the Republic of Korea. BMC Cancer 2014, 14, 420. [CrossRef]

49. Suh, M.; Song, S.; Cho, H.N.; Park, B.; Jun, J.K.; Choi, E.; Kim, Y.; Choi, K.S. Trends in Participation Rates for the National Cancer Screening Program in Korea, 2002-2012. Cancer Res. Treat. 2017, 49, 798-806. [CrossRef]

50. Choi, E.; Lee, Y.Y.; Suh, M.; Lee, E.Y.; Mai, T.T.X.; Ki, M.; Oh, J.K.; Cho, H.; Park, B.; Jun, J.K.; et al. Socioeconomic Inequalities in Cervical and Breast Cancer Screening among Women in Korea, 2005-2015. Yonsei Med. J. 2018, 59, 1026-1033. [CrossRef]

51. Yaffe, M.J. Mammographic density. Measurement of mammographic density. Breast Cancer Res. 2008, 10, 209. [CrossRef]

52. Winkel, R.R.; von Euler-Chelpin, M.; Nielsen, M.; Diao, P.; Nielsen, M.B.; Uldall, W.Y.; Vejborg, I. Inter-observer agreement according to three methods of evaluating mammographic density and parenchymal pattern in a case control study: Impact on relative risk of breast cancer. BMC Cancer 2015, 15, 274. [CrossRef]

53. Bernardi, D.; Pellegrini, M.; Di Michele, S.; Tuttobene, P.; Fanto, C.; Valentini, M.; Gentilini, M.; Ciatto, S. Interobserver agreement in breast radiological density attribution according to BI-RADS quantitative classification. La Radiol. Med. 2012, 117, 519-528. [CrossRef]

54. Lee, E.H.; Jun, J.K.; Jung, S.E.; Kim, Y.M.; Choi, N. The Efficacy of Mammography Boot Camp to Improve the Performance of Radiologists. Korean J. Radiol. 2014, 15, 578-585. [CrossRef] [PubMed]

55. Brandt, K.R.; Scott, C.G.; Ma, L.; Mahmoudzadeh, A.P.; Jensen, M.R.; Whaley, D.H.; Wu, F.F.; Malkov, S.; Hruska, C.B.; Norman, A.D.; et al. Comparison of Clinical and Automated Breast Density Measurements: Implications for Risk Prediction and Supplemental Screening. Radiology 2016, 279, 710-719. [CrossRef] [PubMed]

56. Wacholder, S.; Hartge, P.; Lubin, J.H.; Dosemeci, M. Non-differential misclassification and bias towards the null: A clarification. Occup. Environ. Med. 1995, 52, 557-558. [CrossRef] [PubMed]

57. Brentnall, A.R.; Cuzick, J.; Buist, D.S.M.; Bowles, E.J.A. Long-term Accuracy of Breast Cancer Risk Assessment Combining Classic Risk Factors and Breast Density. JAMA Oncol. 2018, 4, e180174. [CrossRef]

58. Rice, M.S.; Tamimi, R.M.; Bertrand, K.A.; Scott, C.G.; Jensen, M.R.; Norman, A.D.; Visscher, D.W.; Chen, Y.-Y.; Brandt, K.R.; Couch, F.J.; et al. Does mammographic density mediate risk factor associations with breast cancer? An analysis by tumor characteristics. Breast Cancer Res. Treat. 2018, 170, 129-141. [CrossRef]

59. Rice, M.S.; Bertrand, K.A.; VanderWeele, T.J.; Rosner, B.A.; Liao, X.; Adami, H.O.; Tamimi, R.M. Mammographic density and breast cancer risk: A mediation analysis. Breast Cancer Res. 2016, $18,94$. [CrossRef]

(C) 2020 by the authors. Licensee MDPI, Basel, Switzerland. This article is an open access article distributed under the terms and conditions of the Creative Commons Attribution (CC BY) license (http://creativecommons.org/licenses/by/4.0/). 